\title{
LA REFORMA CONSTITUCIONAL DE 2016 Y SUS RAZONES
}

MARCO OLIVETTI 


\title{
LA REFORMA CONSTITUCIONAL DE 2016 Y SUS RAZONES
}

\author{
MARCO OLIVETTI ${ }^{1}$
}

1. El 4 de diciembre de 2016, el cuerpo electoral italiano fue llamado a pronunciarse, por tercera vez en quince años, en un referéndum constitucional, para confirmar o rechazar una ley de revisión constitucional que había sido previamente aprobada por el Parlamento, a principios de 2016. En la votación del referéndum, casi tres electores de cada cinco se expresaron contra la ley de revisión constitucional fuertemente deseada por el Gobierno entonces en el cargo, una coalición de centroizquierda guiada en aquel momento por Matteo Renzi.

El resultado de referéndum de 2016 provocó importantes consecuencias políticas. El Presidente del Consejo de Ministros, Matteo Renzi, dimitió el 7 de diciembre de 2016, como había anunciado varias veces en los meses previos al referéndum, ligando la suerte de su gobierno a la de la reforma constitucional.

Se abrió así un camino político que, quince meses después, llevó a la victoria de las fuerzas políticas populistas en las elecciones del 4 de marzo de 2018 y luego, el 1 de junio, a la formación del primer gobierno populista en una gran democracia de Europa occidental. Pero la votación del 4 de diciembre, además de configurarse como una etapa crucial en la evolución política de Italia (y en algunos aspectos de toda Europa) y además de insertarse en una serie de votaciones anti-establishment que marcaron todo el 2016 (referéndum sobre el Brexit, referéndum sobre acuerdos de paz en Colombia, la elección de Donald Trump a la presidencia de los Estados Unidos de América), ha tenido un significado muy importante para el interminable debate italiano — que se prolonga ya por más de treinta años — sobre una serie de reformas a la Constitución italiana de 1947.

De ese largo —y en buena medida infructuoso- debate, la XVII legislatura republicana (2013-2018) ha constituido por ahora la última etapa, que terminó precisamente con el referéndum del 4 de diciembre de 2016. Se ha tratado, como decía-

${ }^{1}$ Catedrático de Derecho Constitucional en la LUMSA Università - Sede de Roma. Dipartimento di Giurisprudenza, Economia, Politica e Lingue moderne. Via Marcantonio Colonna, 19-00192 Roma. Email:m.olivetti65@gmail.com

Traducción del italiano al español por Carlos Vidal Prado. 
mos, de una conclusión negativa: el proyecto de revisión constitucional discutido durante tres años y luego aprobado por el Parlamento italiano, fue claramente rechazado por los votantes. Dos años y medio después del referéndum de 2016, aún puede ser útil volver brevemente a los contenidos de ese proyecto de reforma, que aspiraba a modernizar la Constitución italiana para conseguir que su sistema de gobierno central fuera más eficiente, haciéndolo menos diferente a los practicados en otros países de la Unión Europea, respecto de los cuales el political decision making system de la República Italiana se ha caracterizado tradicionalmente por una mayor complejidad (y, en consecuencia, por un proceso de toma de decisiones más engorroso). De hecho, tal complejidad se justificó en las décadas posteriores a la Segunda Guerra Mundial por la necesidad de permitir la coexistencia pacífica en el mismo sistema de fuerzas políticas radicalmente heterogéneas (en Italia existía, como se sabe, el mayor Partido Comunista en el mundo libre), pero desde los años ochenta en adelante, una vez superados los hechos (nacionales e internacionales) que dieron origen a esta complejidad, se ha demostrado que carece de justificaciones sociales y políticas.

Los contenidos principales de la ley de revisión debatida por el Parlamento se resumieron en la pregunta del referéndum (quesito referendario) a la que la Oficina central para el referéndum en la Corte di cassazione dio luz verde en agosto de 2016. El texto de la pregunta, que también fue objeto de dos recursos ante el TAR-Lazio, ambos rechazados, era el siguiente: « ¿Aprueba el texto de la ley constitucional concerniente a «disposiciones para la superación del bicameralismo parlamentario, para la reducción del número de parlamentarios, la contención de los costes de funcionamiento de las instituciones, la supresión del CNEL y la modificación del título V de la parte II de la Constitución» aprobado por el Parlamento y publicado en la Gaceta Oficial n. 88 de 15 de abril de 2016?» ${ }^{2}$.

2. Antes de analizar brevemente los contenidos de la reforma constitucional de 2016, quizás sea útil recordar las características principales del largo —y por lo demás no concluyente- debate sobre las reformas constitucionales en Italia ${ }^{3}$, en el que, como dijimos, se insertaba. Se trata de un debate que se prolonga ya a lo largo de cuatro décadas, que puede dividirse en al menos tres fases.

La primera comprende el período que va desde el «Decálogo Spadolini» (1982) hasta las elecciones de 1994, en el que el tema central fue la racionalización del régimen parlamentario, a fin de facilitar su eficiencia y estabilidad, con el objetivo de cumplir la promesa no mantenida de la Asamblea Constituyente con la agenda Peras-

${ }^{2}$ Sobre la pregunta del referéndum de 2016 remito a lo dicho en M.Olivetti, «El eterno retorno de las reformas constitucionales en Italia y de su fracaso: ¿Una Constitución irreformable?», en J.M. Castellá Andreu (coord.), Parlamento, ciudadanos y entes territoriales ante la reforma constitucional: ¿Quién y como participa?, Tirant lo Blanch, Valencia, 2018, p. 76-77.

${ }^{3}$ Sobre este debate, véase la síntesis recogida en M. Olivetti, «Il referendum costituzionale del 2016 e la storia infinita (e incompiuta) delle riforme costituzionali in Italia», en Cuestiones constitucionales, 18 (2008), p. 107-167. 
si, la hoja de ruta aprobada en septiembre de 1946 por la II subcomisión de la Asamblea Constituyente, en la cual la opción a favor de la forma de gobierno parlamentario estaba vinculada al compromiso de corregirla para evitar la degeneración del parlamentarismo. La «agenda» de las reformas institucionales se refería entonces solo a la forma de gobierno - y entre otras cosas a la estructura del bicameralismo- y a la ley electoral, concebida principalmente como un instrumento para racionalizar el régimen parlamentario, frente a la comprobada inestabilidad del Sistema parlamentario italiano en los primeros 35 años posteriores a la entrada en vigor de la Constitución de 1947.

a) Esta primera fase no fue completamente improductiva: aunque la Constitución no se reformó, las leyes electorales se modificaron radicalmente después del referéndum abrogatorio de la ley electoral del Senado celebrado el 18 de abril de 1993 y las leyes n. 276 y 277 de 1993 introdujeron en Italia un sistema predominantemente mayoritario para la elección de la Cámara de Diputados y el Senado de la República. La aplicación de estas leyes, en el contexto de una grave crisis económica, moral e institucional que afectó a Italia a principios de los años noventa del siglo pasado, llevó a la sustitución del sistema de partidos nacido al final de la Segunda Guerra Mundial (y que se conservó casi intacto durante aproximadamente medio siglo), por un nuevo sistema político y una cultura política que también es muy diferente de la dominante en el período comprendido entre 1947 y 1994, hasta el punto de que un agudo observador habló de una verdadera revolución constitucional ${ }^{4}$, mientras que otros constataron el establecimiento de una verdadera «segunda República» ${ }^{5}$. Muchos de los operadores del sistema político se formaron en 1993-94 y no pocos observadores de su dinámica defendieron la necesidad de reformas constitucionales que, modificando la forma de gobierno y el sistema de autonomías territoriales, acompañasen los cambios que se habían producido en el sistema de partidos. Esto abrió una segunda fase en el debate sobre las reformas constitucionales.

b) Dicha fase se inauguró con la declaración de los ganadores de las elecciones de 1994, Berlusconi, Fini y Bossi, que el 1 de abril de 1994 anunciaron su intención de promover una reescritura radical de la Constitución, reformándola en un sentido presidencial y federal. En esta fase, caracterizada por una confrontación política sobre la estructura general de la Constitución de 1947, que se cuestionaba en su conjunto, se desarrollaron varios proyectos basados en una nueva forma de gobierno: piénsese en los proyectos del Comité Speroni en $1994^{6}$, de la Comisión bicameral D’Alema en

${ }^{4}$ C. Fusaro, La rivoluzione costituzionale, Rubbettino, Soveria Mannelli, 1993.

5 G.U. Rescigno, «A proposito di prima e seconda Repubblica», en Studi parlamentari e di politica costituzionale, 1995.

${ }^{6}$ Véase Presidenza del Consiglio dei Ministri, Dipartimento per le riforme istituzionali, Relazione finale del Comitato di Studio sulle riforme istituzionali, elettorali e costituzionali, Istituto Poligrafico e Zecca dello Stato, Roma, 1995. 
$1997-98^{7}$, en la reforma del Título V de la parte II de la Constitución en $2001^{8}$ y en el proyecto de una contrarreforma institucional elaborado por el gobierno Berlusconi entre 2001 y 2005. Esta etapa acabó con la derrota de la reforma constitucional de centro-derecha en el referéndum del 25 y 26 de junio de 2006.

c) La tercera fase coincide con las legislaturas XV, XVI y XVII (respectivamente, 2006-08, 2008-13 y 2013-18), durante las cuales el debate volvió a centrarse en la racionalización de la forma de gobierno y la reforma del bicameralismo (con la adición del tema de la corrección del título $\mathrm{V}$, generado por el boicot y el fracaso de la reforma constitucional de 2001). Desde este perspectiva se sitúa el llamado proyecto Violante, el denominado «ABC constitucional» de la XVI legislatura ${ }^{9}$, el informe de la Comisión de expertos nominados por el Gobierno Letta ${ }^{10}$ y, por último, la reforma constitucional Renzi-Boschi, rechazada, como dijimos, por el electorado el 4 de diciembre de 2016 y cuyo contenido se analizará en las siguientes páginas.

La reforma constitucional de Renzi-Boschi ${ }^{11}$, a pesar de su amplitud en términos del número de artículos modificados (un número alto, también debido a las exigencias de la coordinación del texto impuesta por la reforma del bicameralismo), no fue una «gran reforma» en el sentido utilizado en su tiempo por el politólogo

7 Sobre los trabajos de la Comisión bicameral, véase. V. Atripaldi, R. Bifulco (coords.), La Commissione parlamentare per le riforme costituzionali della XIII legislatura. Cronaca dei lavori e analisi dei risultati, Giappichelli, Torino, 1998.

8 Sobre la reforma del 2001, una de las pocas efectivamente realizadas, existe abundante literatura. Nos limitamos a remitir al primer comentario sobre ella: T. Groppi, M. Olivetti (coords.), La Repubblica delle autonomie. Regioni ed enti locali dopo la riforma del titolo V, Giappichelli, Torino, 2001.

9 Sobre este proyecto, remito a lo escrito en M. Olivetti, «Il vestito di Arlecchino: prime note sul c.d. ABC costituzionale», in www.federalismi.it, 2.5.2012.

${ }_{10}$ Para el informe de dicha Comisión - de la cual el autor de estas páginas ha sido miembro - véase. AA.Vv. (Commissione per le Riforme istituzionali presieduta da G. Quagliariello), Per una democrazia migliore. Relazione finale e documentazione, Dipartimento per l'Informazione e l'editoria - Presidenza del Consiglio dei Ministri (Gangemi editore), Roma, 2013. Véase también A. Cardone (coord.), Le proposte di riforma della Costituzione, Edizioni Scientifiche Italiane, Napoli, 2014.

11 En torno a la reforma constitucional de 2016 se ha publicado una extensa literatura. Entre los diversos comentarios, véase P. Costanzo, A. Giovannelli, L. Trucco (coords.), Forum sul d.d.l. costituzionale «Renzi-Boschi». Dieci studiosi a confronto, G. Giappichelli, Torino, 2015; A. Apostoli, M. Gorlani, S. Troilo (coords.), La Costituzione in movimento. La riforma costituzionale tra speranze e timori, G. Giappichelli, Torino, 2016; E. Rossi, Una Costituzione migliore? Contenuti e limiti della riforma costituzionale, Pisa University Press, Pisa, 2016; Aa.Vv., La riforma della Costituzione. Una guida con le analisi di 15 costituzionalisti, Corriere della Sera, Milano, 2016; M. Dogliani, Costituzione e antipolitica, CRS/Ediesse, Roma, 2016; G: Crainz, C. Fusaro, Aggiornare la Costituzione. Storia e ragioni di una riforma, Donzelli, Roma, 2016; V. Onida, G. Quagliariello, Perché è saggio dire No. La vera storia di una riforma che ha "cambiato verso», Rubbettino, Soveria Mannelli, 2016; F.S. Marini, G. Scaccia (coords.), Commento sistematico alla riforma costituzionale del 2016, Editoriale Scientifica, Napoli, 2016; M. Cavino, L. Conte, L. Imarisio, S. Sicardi, G. Sobrino, C. Tripodina, La riforma respinta (2014-2016). Riflessioni sul d.d.l. costituzionale Renzi-Boschi, Il Mulino, Bologna, 2017; véase también el número monográfico 2/2016 de la revista Quaderni costituzionali, dedicado a Dieci domande sulla riforma costituzionale. 
Gianfranco Miglio, ya que no habría alterado la estructura básica de la Constitución de 1947 (como, en cambio, habría ocurrido si se hubiera aprobado uno de los proyectos de reforma elaborado en la segunda de las tres fases del debate sobre la reforma mencionado en el párrafo anterior). Era, más bien, un «mantenimiento constitucional extraordinario» ${ }^{12}$ impuesto por la prolongada inercia en la actividad esencial de actualización de un texto constitucional, que es necesaria para preservar su actualidad, rejuveneciéndolo en relación con sus perfiles superados y adaptándolo a los tiempos.

3. El corazón de la ley constitucional aprobada por las dos cámaras en 2016 y rechazada por el electorado era la reforma del bicameralismo.

Esta reforma tuvo sus raíces en el origen mismo del bicameralismo italiano después de la Segunda Guerra Mundial, que había sido elegido en la Asamblea Constituyente de 1946-47 más por défault (como consecuencia de la falta de acuerdo entre las fuerzas políticas sobre otras propuestas) que en ejecución de un proyecto bien diseñado. En 1946-47, de hecho, los partidos de la izquierda marxista, en homenaje a un enfoque jacobino (ya manifestado en el primer constituyente francés de 194546), eran favorables a un Parlamento de cámara única, que debería haber sido el único intérprete de la soberanía popular. Sin embargo, los demócratacristianos eran favorables a un bicameralismo al mismo tiempo diferenciado, por la composición de las dos Cámaras (solo la Cámara de Diputados debería haber sido una expresión del principio de representación política, mientras que el Senado debería haber representado las autonomías territoriales y sociales) y paritario (en lo que respecta a los poderes, que deberían haber sido los mismos que la Cámara), mientras que varias fuerzas menores de centro (Partido Republicano, Partito d'Azione) estaban a favor de construir la segunda Cámara sobre la base del principio territorial.

En este contexto, el bicameralismo se había convertido en uno de los temas más controvertidos en el trabajo de los Constituyentes: después de que el enfoque democristiano prevaleciera en el Proyecto de Constitución elaborado por la Comisión de los 75 (que había previsto un Senado parcialmente elegido por los Consejos Regionales), la Asamblea modificó de manera incisiva la estructura del Parlamento, que se articuló en dos cámaras elegidas ambas por sufragio universal, y que se diferenciaron ligeramente en cuanto a los requisitos del sufragio activo y pasivo, a la duración de su mandato (inicialmente se programó un período de seis años para el Senado, en comparación con los cinco de la Cámara) y al sistema de elección (el Senado debería haber sido elegido a nivel regional). En cambio, la Constituyente no se pronunció sobre la fórmula electoral, que se confió a un orden del día de la propia Asamblea, en el que se prefiguraba la elección de la Cámara con sistema proporcional y la del Senado con circunscripciones uninominales. A las dos cámaras, así configuradas, se les

12 Al respecto, vid. F. Palermo (coord.), La «manutenzione» costituzionale, Cedam, Padova, 2007. 
confirieron los mismos poderes: en la legislación (ordinaria y constitucional) ${ }^{13}$, en la relación de confianza con el gobierno ${ }^{14}$, en el control parlamentario ${ }^{15}$.

La práctica constitucional se encargó a continuación de neutralizar los elementos residuales de diferenciación entre las dos Cámaras, mientras la legislación electoral preveía sistemas proporcionales para ambas Asambleas (aunque el del Senado era ligeramente más selectivo, tanto por el uso de distritos uninominales, como por la base regional) y la ley constitucional n. 2/1963 eliminó la diferencia en la duración del mandato, con la consecuencia de que ahora es para ambas cámaras de 5 años (de hecho, las dos Cámaras siempre se han elegido al mismo tiempo). El resultado fue un extraño bicameralismo-fotocopia, en el que las dos Cámaras han funcionado más como articulaciones de un órgano orientado de manera unitaria y coordinada (el Parlamento) que como componentes distintos y diferenciados de éste. Además, el principal factor que neutralizó el bicameralismo fue, al menos hasta 1992, el sistema de partidos: por estos, más que por la Cámara de Representantes y el Senado entendidos como órganos distintos, votaban los electores, distribuyendo entre ellos las cuotas de poder que les permitían negociar la formación de los gobiernos.

4. Sin embargo, la crisis de la República de los partidos y el paso en 1993 a un sistema electoral predominantemente mayoritario rápidamente pusieron en evidencia los riesgos que inevitablemente conlleva un sistema de bicameralismo paritario basado en la doble confianza. Ya en las elecciones legislativas del 27 y 28 de marzo de 1994, las mayorías en las dos Cámaras no coincidían completamente (el centro-derecha liderado por Silvio Berlusconi tenía, de hecho, la mayoría de los escaños en la Cámara y solo pudo conquistarla en el Senado mediante algunos senadores tránsfugas de la oposición) y en comicios posteriores se reprodujo esta no coincidencia tras las elecciones de 2006 y, en una forma más dramática, en 2013, mientras que en el curso de la legisladura que comenzó en 1996 se verificaron desacuerdos entre las dos asambleas. Más allá de este último caso, se puede observar que en tres elecciones políticas de las seis celebradas en la era del sistema electoral mayoritario (1948-1993), las mayorías de las dos Cámaras no eran coincidentes.

El clímax de este asunto se alcanzó después de las elecciones del 24 y 25 de febrero de 2013, cuando, en presencia de dos sistemas electorales similares pero parcialmente diferentes para la elección de las dos Cámaras (con un premio mayoritario en todo el país a nivel nacional en la Cámara y a escala regional en el Senado, de acuerdo con lo previsto en la Ley n. 270/2005), las Cámaras estaban compuestas con orientaciones políticas diferentes: mientras que en la Cámara de Diputados, la coalición de centro-izquierda obtuvo el 29.6 por ciento de los votos y la mayoría absoluta de los escaños, el Senado estaba compuesto por cuatro grupos principales (Partido Demo-

13 Art. 70 y 138 Constitución italiana.

14 Art. 94 Constitución italiana.

15 Art. 82 Constitución italiana. 
crático y otras fuerzas de centro-izquierda, centro-derecha, Movimiento Cinco Estrellas, Scelta Civica y otras fuerzas de centro). La consecuencia fue la parálisis política, que se manifestó por primera vez en la imposibilidad de que el líder de centro-izquierda Pierluigi Bersani formara un nuevo gobierno y más tarde en el impasse de las elecciones presidenciales, que se resolvió solo con la reelección del entonces presidente Napolitano, de 88 años de edad, el cual promovió la formación de un gran gobierno de coalición, liderado por Enrico Letta, con un programa de reforma orgánica de la Constitución. Las contradicciones del bicameralismo estaban, por lo tanto, en el centro de la reforma constitucional y al comienzo del camino para llevarla a cabo.

5. Por este motivo, se puede argumentar que la superación del bicameralismo paritario era la opción más importante contenida en la reforma constitucional de 2016. Sobre la base de esta opción de fondo, una pluralidad de disposiciones habría diferenciado la posición de las dos Asambleas parlamentarias nacionales, tanto las funciones que globalmente les corresponden (artículo 55), como en términos de participación en el proceso de formación de la ley estatal (artículo 70) como, finalmente, con respecto a la relación fiduciaria con el Gobierno (artículo 94).

Sobre esta base (que podría definirse como su pars destruens), la reforma optó por una reforma incisiva del Senado. Entre las diversas direcciones posibles en las que se podría orientar la nueva configuración de la segunda asamblea parlamentaria nacional, la reforma constitucional de 2016 optó por la calificación del Senado como Cámara en la que estarían representadas las instituciones territoriales, retomando así un proyecto que ya había surgido en la Asamblea constituyente y propuesto repetidamente en diversos proyectos presentados desde los años ochenta en adelante ${ }^{16}$. El «rostro» del nuevo Senado se habría modificado radicalmente con respecto a la tradición de la posguerra: y esto tiene que ver con la composición, con el modo de elección, con las funciones y — por lo que se ha dicho de manera esbozada — con el funcionamiento.

6. El Senado diseñado por la reforma constitucional fallida de 2016 debería haber estado compuesto por 100 senadores (muchos menos que los 315 que conforman el Senado italiano actual), de los cuales 95 deberían haber sido al mismo tiempo titulares de cargos políticos regionales (consejeros regionales) o municipales (alcaldes). De hecho, los senadores se habrían distribuido entre las distintas Regiones y provincias autónomas, garantizando un umbral mínimo de dos senadores para cada Región o provincia autónoma (un alcalde y un consejero regional). Las Regiones más pobladas habrían tenido un número adicional de senadores —elegidos entre los consejeros regionales - en proporción a la población regional. En total, el Senado habría estado

16 Sobre este problema remito a M. Olivetti, I raccordi fra le istituzioni nazionali e quelle regionali e locali: i problemi posti dalla trasformazione del Senato in una Camera delle autonomie territoriali, en N. Antonetti, U. De Siervo (coord.), Che fare delle Regioni? Autonomismo e regionalismo nell'Italia di oggi. Atti del Convegno dell'Istituto Luigi Sturzo, Roma, 24-25 gennaio 2014, Istituto Luigi Sturzo/Rodorigo Editore, Roma, 2014, p. 219-264. 
compuesto por 74 senadores-consejeros regionales y 21 senadores-alcaldes, a los cuales se les debían agregar 5 senadores designados — como en el texto constitucional actual, pero con un mandato ya no vitalicio, sino limitado a siete años- por el presidente de la República entre los ciudadanos que hayan «ilustrado a la patria por los más altos méritos en los campos social, científico, artístico y literario» (actual artículo 59 de la Constitución).

Un Senado compuesto por políticos locales electos habría sido coherente con la configuración de la asamblea de Palazzo Madama como la Cámara encargada de representar a las instituciones territoriales: esta función se habría visto facilitada por el hecho de que los senadores habrían sido al mismo tiempo miembros de los consejos regionales o alcaldes.

7. La competencia para la elección de los senadores, que según la Constitución italiana pertenece al cuerpo electoral (rectius: a la parte de este último compuesta por ciudadanos mayores de veinticinco años), habría sido atribuida por la reforma de 2016 - para 95 senadores de un total de 100_ a los Consejos Regionales y a los Consejos de las Provincias Autónomas. Se debe enfatizar que estos órganos habrían sido competentes para elegir no solo a los senadores-consejeros regionales, sino también a los senadores-alcaldes. De los órganos representativos regionales y las provincias autónomas, se derivarían 95 senadores de los 100 (como ya se mencionó, los otros cinco serían nombrados, como hoy, por el Presidente de la República).

La elección de los senadores se llevaría a cabo al comienzo del mandato de cada Consejo regional o provincial, en relación con los senadores (consejeros regionales y alcaldes) de cada Región. Como resultado, no habría habido elecciones generales para el Senado, a excepción de la primera elección después de la entrada en vigor de la reforma constitucional (que, como hemos visto, nunca ha ocurrido). En las elecciones posteriores a la primera, el Senado habría sido renovado proporcionalmente por cada Consejo regional después de su elección. Además, como cada senador tendría que permanecer en el cargo durante un período correspondiente al mandato del Consejo que lo habría elegido, pero a condición de mantener su condición de legitimación de la elección (consejero regional o alcalde), habría sido necesario elegir nuevamente al senador-alcalde cada vez que el senador hubiera dejado de ser alcalde.

8. Con respecto al funcionamiento del Senado previsto por la reforma, esta última habría confirmado, por un lado, las características tradicionales de la cara "parlamentaria» de la Segunda Cámara italiana: la exclusión del mandato impertativo (artículo 67 de la Constitución), irresponsabilidad por las opiniones expresadas y los votos emitidos, la necesidad de autorización para proceder a la limitación de las libertades personales, de domicilio y de la correspondencia (artículo 68 de la Constitución). Sin embargo, se habría previsto un régimen diferenciado respecto al previsto para los diputados en lo que se refiere a la remuneración de sus componentes. 
Al mismo tiempo, la reforma fue señalada por aquello que no preveía: no habría aceptado los rasgos característicos del modelo alemán de la segunda cámara, como las instrucciones vinculantes y el voto unitario por delegación parlamentaria regional. Sin duda, algunos críticos dedujeron que, por estas razones, el Senado no podía funcionar como una Cámara de las autonomías territoriales, pero esta conclusión fue excesiva y se explica — como muchas críticas a la reforma- con el espíritu polémico de la campaña electoral de 2016: por lo demás, el Bundesrat germánico es un modelo a su manera único, nunca imitado por completo por ninguna de las Constituciones que se inspiraron en él (piénsese en la Constitución de Sudáfrica de 1996). Por lo tanto, la posibilidad de que el Senado descrito por la reforma de 2016 funcionara realmente como una Cámara de las autonomías territoriales, capaz de llevar el punto de vista de las Regiones y Municipios al procedimiento legislativo estatal, encontró en la reforma constitucional de 2016 las condiciones estructurales básicas que habrían permitido la concreción efectiva, sobre todo por el hecho de que los miembros del Senado habrían sido, al mismo tiempo, cargos electos locales. Precisamente esta condición — superficialmente criticada por muchos con el ridículo argumento que interpretaba el Senado como una especie de «trabajo complementario» («dopolavoro») para los consejeros regionales y alcaldes- era la piedra angular de una reforma que apostó por la posibilidad de que el Senado se convirtiera en el anillo de conjunción entre el sistema de las autonomías y el proceso de formación de la ley estatal.

En cuanto a la crítica, formulada por algunos, según lo cual habría sido muy difícil para los consejeros regionales y los alcaldes elegidos senadores ejercer ambos mandatos (el de administrador local o de consejero regional y el de miembro de la Segunda Cámara), también era superficial, ya que olvidaba todos los casos de doble mandato conocidos en el derecho comparado. Se habría tratado, como es evidente, de una cuestión de organización. El ejercicio eficaz del doble mandato dependería de la capacidad del Senado diseñado en la fracasada reforma de 2016 para organizarse de una manera completamente diferente a la del Senado previsto en la Constitución de 1947 y aún operativa, para llevar a cabo en muy poco tiempo las tareas (para nada marginales) que la reforma constitucional de 2016 le asignaba, y hacerlo de modo que no impidiese que sus miembros desempeñasen de modo eficaz el mandato «principal» (el de consejero regional o alcalde) del cual dependía la condición de senador. Pero juzgar el Senado descrito por la reforma constitucional de 2016 teniendo en mente la forma en que funciona el actual Senado en virtud de la Constitución italiana fue una operación intelectualmente desordenada: la discontinuidad entre los dos modelos del Senado era muy clara, similar a la que se produjo entre el Senado Real y el Senado Republicano después de la Segunda Guerra Mundial.

Los problemas de funcionamiento de la «Cámara alta» situada en el centro de la reforma de 2016 deberían haber sido juzgados con un enfoque cultural capaz de captar todas las novedades aportadas por la reforma: faltó una capacidad de lectura de este tipo, sin embargo, en la parte de la doctrina italiana que criticó duramente la 
reforma y, especialmente, en los eruditos de mayor edad y más conservadores, que, en su mayor parte, malinterpretaron su significado y alcance.

9. Las funciones atribuidas al Senado fueron objeto de una lista detallada en el art. 55.5 del texto constitucional, modificado por la reforma, y a las que se indican en el mismo, debían agregarse después las resultantes de otras disposiciones constitucionales, allí donde los mecanismos previstos en ellas no fuesen atribuibles a las funciones enumeradas en el art. 55.

En este contexto, la participación en el ejercicio de la función legislativa habría continuado siendo una de las funciones más importantes del Senado que habría surgido de la reforma. Esta participación debería haber tenido lugar de diferentes maneras, de acuerdo con los cuatro procedimientos legislativos descritos en el art. $70^{17}$.

a) En primer lugar, el Senado habría mantenido, en un nivel de plena paridad con la Cámara de Diputados, la competencia para aprobar las leyes en los asuntos enumerados en el primer párrafo del art. 70. Se trataba de un elenco significativo, que incluía, además de las leyes constitucionales, también una serie de leyes ordinarias relativas a las autonomías territoriales y al propio Senado, en materia de proceso de integración europea y de ejecución de instituciones de democracia directa. La lista era obviamente discutible desde un punto de vista analítico, pero parecía compartible en cuanto inspirada en la idea de excluir del área de las «leyes bicamerales» las leyes relacionadas con las grandes políticas públicas (que, en cambio, estarían dentro del alcance del procedimiento general, del que se hablará a continuación) y de confiar a la competencia paritaria de las dos Cámaras únicamente las leyes ordinarias, que hubieran establecido las reglas del juego de las relaciones entre los diferentes niveles de gobierno (Estado, Unión Europea, autonomías territoriales).

En todos los demás casos, la participación del Senado en el proceso de formación de las leyes ya no habría tenido lugar en igualdad de condiciones, pues la Cámara alta se habría colocado en una posición de subordinación respecto a la Cámara de Diputados, elegida por sufragio universal. Esta, por lo demás, es la condición en la que se ubican las segundas Cámaras de los parlamentos de todos los estados europeos (con la única excepción, hasta hoy, de nuestro país y Suiza): la del bicameralismo no paritario, sino asimétrico.

b) La regla general del proceso de formación de la ley, según la reforma, habría sido la regulada por el art. 70, párrafo 3, del texto que resultaría de la reforma: una vez aprobado por la Cámara de Diputados, un texto legislativo debía trasladarse al Senado, que podría decidir examinarlo y para este fin habría sido suficiente la solici-

17 Sobre este tema remito a lo expuesto en M. Olivetti, I procedimenti legislativi (art. 70-74), en F.S. Marini, G. Scaccia (coords.), Commento sistematico alla riforma costituzionale del 2016, Editoriale Scientifica, Napoli, 2016. 
tud de un tercio de los senadores, que debía presentarse dentro de los diez días siguientes a la transmisión del texto legislativo. Una vez formulada esta solicitud, el Senado habría tenido un mes para examinar el texto aprobado por la Cámara de Diputados y formular, si lo hubiera considerado apropiado, propuestas de modificación. Estas debían ser transmitidas a la Cámara de Diputados, a la cual habría correspondido la decisión final: podría aceptar las modificaciones propuestas por el Senado o mantener el texto original. En ambos casos, el Jefe de Estado podría proceder a la promulgación y publicación de la ley. Una extensión de los tiempos de los procedimientos parecía concebible solo en el caso de que la Cámara de Diputados hubiese aprobado, durante la segunda deliberación, nuevas enmiendas al texto legislativo, que en este caso deberían haber sido enviadas al Senado, el cual podría haber ejercido sobre ellas el poder de reclamarlas. O en el caso de que el Presidente de la República hubiese remitido nuevamente al Parlamento (sobre la base del poder de veto suspensivo que le pertenece en virtud del artículo 74 de la Constitución) la ley aprobada de acuerdo con el procedimiento que hemos visto.

c) Con respecto al esquema procedimental ahora descrito brevemente, los párrafos 4 y 5 del art. 70 preveían después dos variantes bien identificadas: la de la Ley de Presupuestos y la de una Ley adoptada en el ejercicio de la cláusula de supremacía, a saber, el poder que la reforma habría reconocido a la ley estatal, para intervenir, con objeto de proteger el interés nacional, incluso fuera de las materias sobre las cuales se reconoce la competencia del Estado. Los procedimientos del cuarto y quinto párrafos del art. 70 de la reforma de 2016 intentaban reconocer al Senado una participación más intensa en el proceso de formación de la ley estatal en comparación con la posible en vía ordinaria de conformidad con el art. 70, apartado 3, pero sin llegar a otorgar a la Camara de las autonomías territoriales un verdadero y propio poder de veto, como habría ocurrido en el caso del procedimiento legislativo bicameral (y como sucede con todas las leyes del sistema constitucional no reformadas y hasta ahora vigente).

10. Con respecto a lo previsto en la fracasada reforma de 2016 para la reducción del número de parlamentarios, la contención de costes de las instituciones y la supresión del Consejo Nacional de la Economía y el Trabajo (CNEL), es posible limitarse a algunas breves observaciones.

El número de parlamentarios electivos se habría reducido incisivamente, de 945 (630 diputados más 315 senadores) a 725 (630 diputados más 95 senadores electivos). Pero la reducción hubiera sido en realidad más amplia: los 95 senadores electivos, de hecho, también habrían sido titulares de otro cargo (el de consejero regional o alcalde) y no habrían recibido más emolumentos que los que les correspondían en virtud de este cargo. De ello se deduce que la reducción en el número de cargos políticos electivos habría sido igual al número de senadores electivos actuales (es decir, trescientos quince). 
De esta información, obviamente se habría derivado una reducción en los costes de funcionamiento de las instituciones, que se habría acentuado por una previsible reducción en los costes del Senado, dado el nuevo rol asignado constitucionalmente a esta asamblea. A esto se añade el ahorro que habría resultado de la supresión del CNEL y lo que habría seguido a la eliminación de los reembolsos a los Consejos Regionales. El sentido de estas medidas debe ser apreciado adecuadamente, sin caer en populismos fáciles que identifican en la reducción del número de parlamentarios la panacea de todos los males, pero captando la señal de la exigencia de una mayor sobriedad de la política que estas medidas pretendían lanzar.

En cuanto a la supresión del Consejo Nacional de la Economía y el Trabajo, debe recordarse que este órgano fue la traducción organizativa débil e infeliz de una idea con gran potencial. De hecho, el CNEL fue en el diseño de los Constituyentes la punta del iceberg de la aspiración a reconocer un estatus constitucional a las principales manifestaciones del pluralismo en la vida económica (las categorías productivas). Lo cierto ahora es que no logró ganarse un papel adecuado para expresar esta instancia y, por lo tanto, la propuesta de suprimirla parecía justificada. Sin embargo, la necesidad para la cual se previó conserva su valor y sigue siendo un tema constitucional abierto, incluso después del fracaso de la reforma y la supervivencia del CNEL y su sustancial inutilidad.

11. La reforma de 2016 también tenía la intención de intervenir sobre el Título $\mathrm{V}$, la parte de la Constitución relativa a las autonomías territoriales, ya completamente reescrita por dos reformas constitucionales diferentes entre 1999 y 2001: en 2016 se intentó una reestructuración general, centrada en la redefinición de la división de las competencias legislativas entre el Estado y las regiones, así como en la simplificación del sistema de autonomías territoriales en su conjunto.

Este objetivo de simplificación del sistema debería haberse logrado mediante la desconstitucionalización de las Provincias (es decir, su eliminación del texto constitucional), dejando así a las fuentes (estatales y regionales) de rango infraconstitucional la potestad de continuarlas previendo o suprimirlas. Así, la reforma constitucional ofreció cobertura constitucional a las reformas legislativas del gobierno Monti ${ }^{18}$ y a la ley Del Rio (ley n. 56/2014) que transformó las provincias en entidades de segundo grado.

Con respecto a la división de competencias legislativas entre el Estado y las Regiones, las principales novedades contempladas por la reforma fallida de 2016 habrían sido las siguientes:

18 Sobre estas reformas remito a M. Olivetti, Las asignaturas pendientes del regionalismo italiano, en la época de la crisis, en E. Alvarez Conde (coord.), El Estado autonómico hacia 2020, Editorial Universidad Rey Juan Carlos, Madrid, 2013, p. 87-120 y a M. Olivetti, Centralization and decentralization: the current trends in Italy, en K. Göymen e O. Sazak (coord.), The Centralization and Decentralization Debate Revisited, Istanbul Policy Center/Stiftung für die Freiheit, Istanbul, 2014. 
a) La eliminación de la competencia legislativa concurrente, que ha caracterizado el regionalismo italiano desde el principio y que, con un exceso de prisa, se ha considerado la responsable de la falta de claridad en la división de roles prevista en la Constitución para Estado y Regiones.

b) La transferencia a la legislación estatal exclusiva de 26 nuevas materias respecto al 2001, delineando un marco de indudable re-centralización legislativa.

c) El mantenimiento de la potestad residual de las Regiones, para todas las materias no atribuidas expresamente a la ley estatal.

d) Enumeración indicativa de algunas competencias legislativas regionales, en el ámbito de una competencia general del Estado para establecer disposiciones generales y comunes.

e) La facultad del legislador estatal a intervenir también «fuera de materia», es decir, en las materias cuya competencia resida en la Región, cuando esto sea requerido por razones de interés nacional.

De este sumario elenco resulta clara la dirección global de la reforma de 2016 en materia de autonomías territoriales, y especialmente de las relaciones entre el Estado y las regiones: era la de una clara re-centralización legislativa. La «mano dura» de los aspirantes a reformadores de 2016, que no habían resistido la tentación de redefinir la división global de competencias legislativas entre el Estado y las Regiones, en lugar de limitarse a una corrección puntual — solicitada por muchos— de diversos aspectos de la reforma de la 2001, generó no pocas perplejidades. Una solución más cautelosa y prudente habría sido sin duda preferible. Pero incluso aquellos que se situasen - como nos parece necesario - en esta perspectiva al menos parcialmente crítica, no podrían haber dejado de incluir esta crítica en un contexto más amplio, que llevaba a relativizarla.

Tres datos deben, en nuestra opinión, ser considerados.

a) El primero es que la re-centralización que sin duda caracterizaba la reforma constitucional de 2016 se limitaba a la legislación y no se extendía a la administración, que se rige por el principio de subsidiariedad, el cual no habría sido modificado por la reforma constitucional: si la reforma hubiera sido aprobada, se habría mantenido la regla en virtud de la cual las funciones administrativas deben ubicarse, en la medida de lo posible, en el nivel de gobierno más cercano al ciudadano (artículo 118.1 de la Constitución).

b) El segundo dato es que la re-centralización legislativa que la reforma hubiera producido sin duda hubiera sido notable comparando el texto de la reforma con el texto constitucional vigente, es decir, con la reforma de 2001. Sin embargo, es ampliamente conocido que este texto se ha vaciado en gran parte en su aplicación: desatendido ab initio por el legislador estatal, que nunca lo ha considerado como un 
verdadero límite a sus competencias y, en cambio, ha continuado legislando etsi titulus quintus non daretur. Además, la reforma del Título $\mathrm{V}$ nunca ha sido tomada en serio por la jurisprudencia constitucional, cuyas directrices, después de alguna tímida apertura inicial (por ejemplo, la sentencia 282/2002), se han asentado en la línea de un consolidado antirregionalismo, renunciando a proteger las atribuciones legislativas regionales y reconociendo a la ley estatal varios títulos de competencia más allá de los que resultan de una lectura rigurosa del texto constitucional (por ejemplo los mecanismos de la «llamada de subsidiariedad» y las numerosas y variadas competencias transversales del legislador estatal).

Finalmente, la reforma de 2001 se enterró definitivamente después de 2010, debido a la crisis económica y en nombre de la necesidad de reducir o limitar el gasto público, que encontraron en la competencia estatal en materia de coordinación de las finanzas públicas (Artículo 119, apartado 2 de la Const. It.) la vía para devolver a la ley estatal la competencia de la competencia. En resumen: el título V de 2001 ya era un montón de escombros incluso antes de la reforma de 2016 y resulta serlo incluso después de que haya sido rechazada por el electorado. La «mano dura» del legislador de revisión, por lo tanto, habría intervenido en una batalla ya perdida para las Regiones, como lo demuestra la pobre resistencia de las propias Regiones ante una operación política de fuerte reducción de su autonomía legislativa.

c) El tercer dato está representado por la «compensación» para las Regiones que, si se hubiera aprobado la reforma, podría haber sido representada — repecto a la pérdida de competencias legislativas (por lo demás, como se mencionó anteriormente, previstas ahora solo en papel)_ por la reforma del Senado y su transformación en una Cámara representativa de las instituciones territoriales, en la que el peso de las Regiones habría sido ampliamente mayoritario (74 senadores de 100 habrían sido consejeros regionales). El trade off que la reforma delineaba, en resumen, era el que se encontraba entre un indudable (y en parte ciertamente criticable, al menos por su amplitud) debilitamiento del espacio para la autonomía legislativa regional y el reconocimiento de un espacio cualificado para las Regiones que hubiera permitido a «sus» senadores participar en la formación de la ley estatal, es decir, lo que la reforma constitucional de 2016 consideraba como el acto normativo por excelencia en la República de las autonomías (la ley estatal, de hecho) y que en el pasado era tradicionalmente un acto de cuya configuración casi siempre «centralista» derivaba una lesión de las autonomías regionales.

En resumen, la reforma apostaba por una legislación estatal negociada con las Regiones a través del Senado, apuntando a esta «válvula» para la recuperación del cariz autonómico del ordenamiento, que inevitablemente se habría perdido en parte debido a la reducción de las competencias legislativas regionales. Dar una voz a las Regiones en la formación de la ley estatal hubiera significado, desde este punto de vista, aplicar esa parte del art. 5 de la Constitución italiana, que obliga a la República a adaptar los criterios y métodos de su legislación a las exigencias de la autonomía y la descentralización. 
12. La reforma constitucional de 2016 no intervenía, por lo tanto, sobre lo que desde los años ochenta del siglo pasado ha sido el tema más discutido en el debate italiano sobre la reforma de la Constitución: la forma de gobierno. Obviamente, la estructura de la forma de gobierno estatal sin duda se habría visto influenciada por las reformas relacionadas con el bicameralismo analizado anteriormente, así como por aquellas relacionadas con el proceso de formación de la ley y por una serie de reformas menores (relacionadas con los decretos-leyes y a la denominada «vía preferencial» para los proyectos de ley declarados de particular importancia por el Gobierno). En general, la reforma del bicameralismo habría fortalecido la posición del gobierno y su mayoría en la Cámara, pero la cuestión del sistema de gobierno, a saber, la racionalización del régimen parlamentario, no se abordó en la reforma constitucional de 2016 y se confió a la reforma del sistema electoral, a través de la ley electoral para la Cámara de Diputados efectivamente aprobada por las dos Cámaras durante el período 2014-2015 (ley n. 52/2015, denominada Italicum). Entre otras cosas, la ley electoral solo regulaba el sistema de elección de la Cámara de los Diputados, presuponiendo que la reforma del Senado contenida en la ley de reforma constitucional no hubiera hecho ya necesario el voto de confianza de este último al Gobierno y que, por lo tanto, el ejecutivo habría podido basarse solo en la mayoría de la Cámara baja, como ocurre en todos los regímenes parlamentarios.

La ley electoral de $2015^{19}$ preveía un sistema proporcional con la asignación de escaños entre las listas concurrentes, correspondiente a los partidos políticos, pero incluyó un "premio de mayoría» a favor de la lista que hubiera obtenido al menos el 40 por ciento de los votos. En el caso de que ninguna lista hubiese alcanzado el 40 por ciento de los votos, la ley electoral de 2015 preveía una segunda vuelta entre las dos listas que obtuvieran el mayor número de votos, con la asignación de un premio mayoritario de 340 escaños (sobre 630) en beneficio de la lista que hubiese vencido a escala nacional. Era un sistema muy criticado por los opositores a las reformas de Renzi-Boschi, quienes entendían que creaba un sistema de gobierno dominado por el Primer Ministro y por la mayoría parlamentaria que se produciría mediante la asignación del «premio de mayoría» otorgado por la aplicación de la ley n. 52/2015. Precisamente por este motivo, la misma reforma constitucional de 2016 contenía algunas reglas inspiradas en el propósito de fortalecer los contrapesos en el circuito gobierno-mayoría: la reforma del sistema de elección del Presidente de la República (para el cual ya no sería posible, ni siquiera después del tercer escrutinio, una elección por mayoría absoluta, sino que habrían sido siempre necesarios a menos tres quintos de los votantes), la apertura a un fortalecimiento de las instituciones de democracia directa, el control previo de la constitucionalidad sobre las leyes electorales (aplicable a la misma ley n. 52/2015), el reconocimiento de un estatuto de los derechos de las

19 Sobre tal reforma remito a M. Olivetti, La reforma electoral italiana de 2015, en Cuadernos de Pensamiento político - FAES, 47 (julio-septiembre 2015), p. 35-52 
minorías y de la oposición en el Parlamento, así como un fortalecimiento de las instituciones de participación popular, que por lo demás todavía está solo esbozado.

El resultado del referéndum constitucional de 2016 ha barrido no solo la reforma constitucional sino también la electoral. Esta última fue declarada inconstitucional por la Corte Costituzionale con la discutible sentencia n. 35/2017, en la cual el juez de las leyes consideró que el «premio de mayoría» previsto por la ley n. 52/2015 no era compatible con la Constitución. Por este motivo, en el segundo semestre de 2017, el Parlamento aprobó una nueva ley electoral (Ley n. 165/2017), de carácter prevalentemente proporcional (pero con algunos elementos mayoritarios), sobre cuya base se celebraron las elecciones legislativas del 4 de marzo de 2018.

13. Dejando de lado ahora la cuestión de la ley electoral, la reforma constitucional de 2016, por lo tanto, presentó varios aspectos positivos.

La reforma más importante fue la del sistema bicameral, que no solo habría resuelto el añejo problema de la existencia de un Senado suspendido entre el papel de la duplicación inútil y el de agente paralizante de la democracia parlamentaria (una verdadera y propia bomba de relojería colocada en el corazón de la Constitución), sino que también habría transformado la Cámara alta en una cámara de autonomía territorial, dando a esta última, y especialmente a las regiones, una voz en el proceso de formación de la ley estatal.

Eran también apreciables las medidas destinadas a contener los costes de las instituciones representativas.

Menos valiosa, sin embargo, era la reforma del regionalismo, demasiado marcada por un espíritu centralista que no estaba suficientemente meditado (o del cual, por el contrario, se esperaban resultados milagrosos), pero que, por otro lado, intervenía sobre una estructura existente que en la realidad se había revelado (y aún lo es) poco funcional y que hoy parece desgastada, con la consecuencia de que incluso un «reinicio» no exento de excesos, como el contenido en el art. 117 reformulada por la reforma de 2016, podría verse como una oportunidad para comenzar una nueva fase.

De hecho, el punto más débil de la reforma consistió en un dato social y político que estaba fuera de ella y que todavía está presente en la sociedad italiana: la crisis de autonomía de los cuerpos intermedios (autonomías sociales y territoriales), que había sido uno de los pilares principales del pensamiento de los Padres Constituyentes y que parece hoy perdida tanto en la cultura política italiana prevalente (tanto de matriz populista, como de diversa inspiración), como en la realidad social, en la cual se registra en los últimos decenios una grave crisis de todos los sujetos sociales organizados, desde los partidos a los sindicatos hasta las diversas formas de asociacionismo. Asimismo, la crisis de legitimación de las autonomías territoriales debe interpretarse en el contexto de una crisis más general de los cuerpos intermedios. La reforma de 2016 era, al mismo tiempo, una expresión de esa crisis y un intento no del todo adecuado para abordarla, al menos en lo que respecta al sistema de autonomía territorial. Esta crisis permanece abierta más de dos años después del referéndum de 2016 y se agrava 
aún más con la llegada al poder de los partidos populistas, por su propia naturaleza no inclinados a la mediación con los cuerpos sociales organizados. Esto requiere que las clases dirigentes, y en general el pueblo italiano, conserven el sentido de la historia y la conciencia de la complejidad del país, que no puede ser gobernado sin una atención continua y paciente a su diversidad.

Title:

The constitutional reform of 2016 and its reasons

\title{
Resumen:
}

Se contiene en este texto un breve análisis sobre la reforma constitucional rechazada en referéndum en Italia, en el año 2016. La reforma más importante fue la del sistema bicameral, que pretendía aclarar el papel del Senado y convertirlo en una Cámara de representación territorial. Esto habría servido tambien para modificar el modelo de relación entre Estado y regiones. Tambié se intentaban reducir costes del funcionamiento de las instituciones representativas. A pesar de que la reforma constitucional fracasó, en el artículo se defienden algunos elementos valiosos de la reforma, que al no haberse aprobado han provocado que, en este momento, siga habiendo disfunciones en el sistema político constitucional italiano.

\begin{abstract}
:
A brief analysis on the constitutional reform rejected in referendum in Italy (in 2016) is contained in this text. The most important reform was the bicameral system, which sought to clarify the role of the Senate and turn it into a Chamber of territorial representation. This would have served also to modify the model of relationship between State and regions. Also is trying to reduce costs of the functioning of representative institutions. While constitutional reform failed, in the article are defended some assets of the reform, which not have been approved have caused that, at this point, continue having dysfunctions in the Italian constitutional political system.
\end{abstract}

Palabras clave:

Reforma Constitucional; Bicameralismo; Senado; Regionalismo

Key words:

Constitutional Amendment; Bicameralism; Senate; Regionalism 